\title{
Trait Anxiety as a Risk Factor for Impulse Control Disorders in de novo Parkinson's Disease
}

\author{
Pauline Waskowiak $^{\mathrm{a}, 1}$, Vincent Koppelmans ${ }^{\mathrm{b}}$ and Marit F.L. Ruitenberg ${ }^{\mathrm{a}, \mathrm{c}, *}$ \\ ${ }^{a}$ Department of Health, Medical and Neuropsychology, Leiden University, Leiden, the Netherlands \\ ${ }^{\mathrm{b}}$ Department of Psychiatry, University of Utah, Salt Lake City, UT, USA \\ ${ }^{\mathrm{c}}$ Leiden Institute for Brain and Cognition, Leiden, the Netherlands
}

Accepted 17 November 2021

Pre-press 7 December 2021

\begin{abstract}
.
Background: In addition to the well-known motor symptoms, patients with Parkinson's disease (PD) also frequently experience disabling non-motor symptoms including impulse control disorders (ICDs). ICDs are characterized by a loss of voluntary control over impulses, drives, or temptations regarding excessive hedonic behavior.

Objective: The present study examined whether depression and anxiety in de novo PD patients predict the prospective development of ICDs.

Methods: We selected 330 de novo PD patients from the Parkinson's Progression Markers Initiative database who were free of ICDs at the start of the study. ICD presence at baseline and follow-up assessments was evaluated via the shortened version of the Questionnaire for Impulsive-Compulsive Disorders (QUIP-S). Baseline depression and anxiety were measured via the Geriatric Depression Scale (GDS-15) and State-Trait-Anxiety Inventory (STAI-Y), respectively.

Results: A total of 149 participants (45.2\%) developed an ICD at follow-up and average time of ICD onset was 35 months after baseline. Results of a Cox regression analysis showed that STAI-Y scores but not GDS-15 scores significantly predicted ICD presence. Specifically, scores reflecting higher trait anxiety were associated with an increased risk of developing an ICD. This effect was not confounded by age, gender or UPDRS motor score. We also replicated the well-established result that dopamine agonist use is predictive of ICDs.

Conclusion: Our findings indicate that higher anxiety levels in de novo PD patients represent a risk factor for ICD development during the course of the disorder. This highlights the need for early and routine based anxiety screening in these patients.
\end{abstract}

Keywords: Anxiety, depression, impulsivity, impulse control, Parkinson's disease

\footnotetext{
${ }^{1}$ Present address: Department of Medical Psychology, Amsterdam Neuroscience, Amsterdam UMC, Vrije Universiteit, Amsterdam, the Netherlands.

*Correspondence to: Marit Ruitenberg, Department of Health, Medical and Neuropsychology, Faculty of Social and Behavioural Sciences, Leiden University, P.O. Box 9555, 2300 RB Leiden, the Netherlands. Tel.: +31 71527 6341; E-mail: m.f.l.ruitenberg@ fsw.leidenuniv.nl.
}

\section{INTRODUCTION}

Parkinson's disease (PD) is a neurodegenerative disorder primarily characterized by clearly observable motor symptoms, such as bradykinesia, resting tremor, and postural instability [1]. In addition to these well-known motor symptoms, people with $\mathrm{PD}$ also frequently experience disabling non-motor 
symptoms, such as autonomic dysfunctions, cognitive impairments, and neuropsychiatric problems [2]. Among these non-motor symptoms are so-called impulse control disorders (ICDs), which occur in up to $20 \%$ of people with PD [3]. ICDs include compulsive, repetitive, and excessive behaviors most commonly in relation to gambling, sexuality, buying and eating. Furthermore, patients might display repetitive and purposeless behaviors (i.e., punding) and/or spend an excessive amount of their time on pursuing specific hobbies (i.e., hobbyism). All of these behaviors are characterized by an inability to resist impulses and the loss of voluntary control over these, negatively affecting quality of life in patients [3, 4]. ICDs are often accompanied by serious secondary consequences, such as financial and psychosocial difficulties which additionally increase the burden on patients and family members [4].

Prior research has shown that dopaminergic replacement therapy (DRT) and in particular the use of dopamine agonists (DAs) increases the risk for ICD development [5-8]. However, much less is known about the contribution of psychological factors such as depression and anxiety to the development of ICDs in PD, even though these have been linked to impulsivity in other populations $[9,10]$ and often co-occur in PD [11]. In fact, depression and anxiety are the most frequent neuropsychiatric symptoms in people with $\mathrm{PD}$, with prevalence estimates ranging between $30-55 \%$ and $40-56 \%$, respectively [12-14]. While prior work suggests that ICDs in PD are associated with higher depression levels $[15,16]$, the causal aspect of this relationship remains largely unknown due to the cross-sectional nature of most prior studies [17]. Recently, a longitudinal study demonstrated that the presence of a clinical depression around the time of PD diagnosis increases the risk for ICDs later in the disease, indicating that depression precedes the occurrence of ICDs [18]. Consistent with the abovementioned findings regarding DRT, the researchers also found DA use to be associated with an increased risk for ICD development. These effects seem to be additive, suggesting that patients who receive DA treatment and experience depressive symptoms are at particularly high risk for the development of ICDs [18]. In addition to depression, anxiety has also been associated with ICDs in PD [19-21]. For instance, two cross-sectional studies reported that anxiety levels were significantly higher in PD patients with ICDs compared to those without [21, 22]. However, even though this general association between anxiety and ICDs has been demonstrated, not much can be said about the temporal relationship of this association due to the lack of longitudinal studies.

To date, it thus remains largely unknown whether depression and anxiety occur as a result of ICDs, or rather represent potential risk factors. Establishing the predictive value of these psychological factors for the development of ICDs may be particularly relevant towards early identification of patients at risk for ICDs and targeted treatment of depressive and/or anxiety symptoms at an early stage to reduce risk. This may help patients maintain quality of life by preventing the multiple negative consequences associated with ICDs. Furthermore, it seems likely that these psychological factors additionally interact with dopaminergic medication in the prediction of ICDs. Delaney et al. (2012) [23] developed a conceptual model describing an interaction between biomedical and psychosocial factors in the genesis of ICDs [23]. According to this model, DA treatment functions as a so-called susceptibility multiplier by increasing the susceptibility that is associated with factors such as depression, anxiety, and stress. When this susceptibility threshold is reached for a certain individual, the patient develops an ICD. A better understanding of this interaction could have essential implications for clinicians and their choice of DRT for people with relatively early-stage PD who additionally experience depression and/or anxiety symptoms.

In the present study we aimed to evaluate whether individual differences in levels of depression and anxiety at baseline could be identified as risk factors for ICD development in patients with PD. Using longitudinal data, we investigated whether self-reported symptoms of depression and anxiety around the time of PD diagnosis can predict the occurrence of ICDs later in the course of the disease. We hypothesized that higher levels of depression and anxiety are significant risk factors for the development of ICDs. This study additionally explored whether the interaction of these psychological factors with dopaminergic medication type, especially DAs, significantly predicts ICDs. This is particularly relevant as literature suggests that mainly DAs - compared to other medication types-are associated with an increased risk for ICDs, yet not much is known about this factor in relation to potential psychological risk factors [1].

\section{METHODS}

The data were retrieved from the Parkinson's Progression Markers Initiative (PPMI) database on May 
4, 2020 and comprised a total of 1,917 enrolled subjects. The PPMI is an international multi-center study with the general objective to identify clinical, imaging, and biological progression markers in patients with PD. The database includes both PD patients (i.e., de novo, prodromal, or with specific genetic mutations) and healthy controls who were repeatedly evaluated at three- or six-months intervals over a period of at least five years. Further details on study goals and design are available in Marek et al. (2011) [24] and via the PPMI website (https://www.ppmiinfo.org). The PPMI study has been approved by independent ethic committees at each clinical site and all participants provided written informed consent prior to participation in the study.

\section{Participants}

Figure 1 shows an overview of the participant selection process for the present study. Criteria for inclusion in the current study were a de novo PD status, a negative ICD screen at baseline, and availability of baseline depression and anxiety measurements. Patients with missing ICD baseline data were excluded from the sample.

\section{Materials and measures}

\section{Impulse control disorders}

The short version of the Questionnaire for Impulsive-Compulsive Disorders rating scale (QUIP-S) was used to assess ICD presence at baseline and follow-up visits. The questionnaire is a self-report screening instrument which assesses the existence of impulsivity and related compulsive behaviors. It assesses the four main ICDs (i.e., gambling, sexuality, buying, and eating) as well as four other compulsive behaviors that may occur in people with PD (i.e., hobbyism, punding, walkabout, and compulsive medication use). All items are presented in a forced-choice format (Yes $=1$ or No $=0$ ) and refer to current behaviors which have been present for at least four weeks. Based on previous research [25], total scores from the first two sections (i.e., main ICDs and compulsive behaviors) were used to identify the presence or absence of an ICD at each time point (score $0=$ no ICD, score 1 or higher = ICD). The QUIP-S was especially designed for the application in people with PD and has been validated in this group [25, 26]. Weintraub and colleagues (2009) [25] demonstrated high sensitivity for the detection of any ICD or related behaviors in

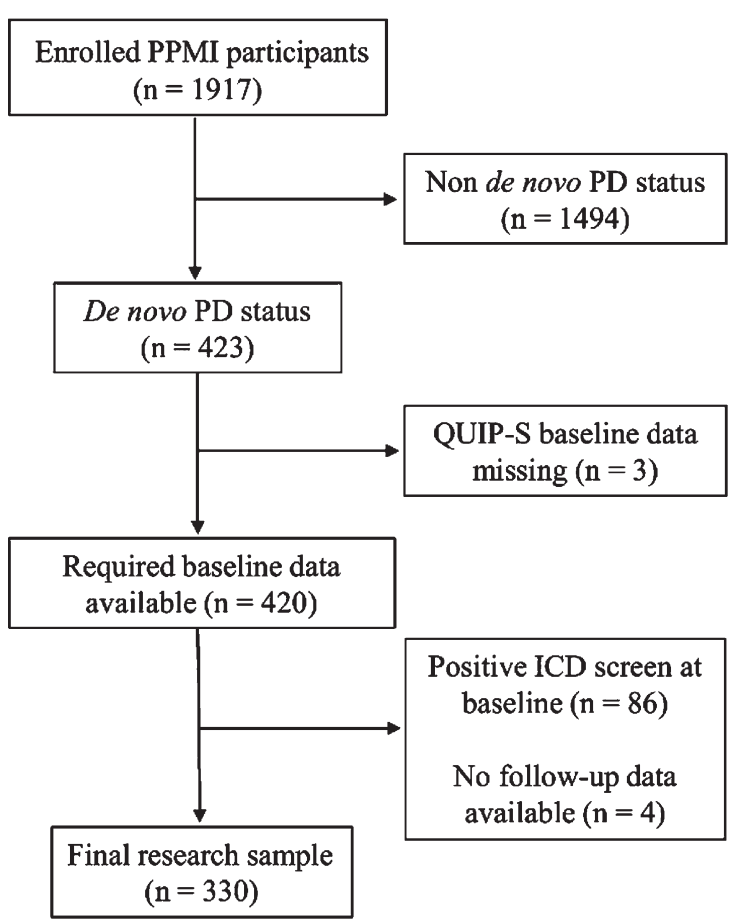

Fig. 1. Flowchart of the participant selection process. ICD, impulse control disorder; PD, Parkinson's disease; PPMI, Parkinson's Progression Markers Initiative; QUIP-S, Questionnaire for Impulsive-Compulsive Disorders, short form.

people with PD (94\%), indicating that the QUIP-S is an adequate screening instrument in this patient population.

\section{Depression}

Depressive symptoms at baseline were assessed via self-reported scores on the 15 -item version of the Geriatric Depression Scale (GDS-15), a frequently used screening instrument to detect depressive symptoms in geriatric patients that has also been validated for people with PD [27]. The questionnaire consists of 15 forced-choice items concerning the emotional state of patients during the past week (e.g., "Do you feel that your life is empty?"). We calculated total scores which could range between 0 and 15 , with higher scores indicating a higher level of depressive symptoms.

\section{Anxiety}

The State-Trait Anxiety Inventory - Form Y (STAI-Y) was used to assess baseline anxiety symptoms in the current study. Although it was initially not designed for PD, the STAI-Y is considered to be a valid instrument for assessing anxiety symptoms 
in people with early-stage PD [28, 29]. The questionnaire measures anxiety in adults on two 20 -item subscales; the state subscale assesses temporal anxiety by asking patients to indicate how they feel at that particular moment (e.g., "I feel frightened"), whereas the trait subscale assesses general anxiety by asking patients to indicate how they generally feel (e.g., "I feel like a failure"). For the present study, we calculated total STAI-Y scores (ranging between 40-160) as well as State- and Trait subscale scores (each ranging between 20-80). Higher scores indicate a higher level of anxiety.

\section{Dopaminergic replacement therapy type}

Medication use was assessed at each visit and any changes in treatment between visits were registered. Based on this information, we categorized the DRT type (DA vs. levodopa/other) for each patient at each visit. This allowed us to consider DRT type as a binary time-dependent covariate in our analyses.

\section{Procedure}

To ensure eligibility for participation in the PPMI study, patients completed a comprehensive screening procedure prior to the baseline measurement. The screening procedure included medical history assessments, physical and neurological examinations (e.g., the UPDRS), dopamine transporter imaging, and several clinical laboratory assessments. Participants who met eligibility criteria were subsequently assessed at baseline (within 45 days) and from then every three months during the first year, and every six months until the fifth year. After that, assessments took place annually. Each visit lasted approximately eight hours and included similar examinations to those that were conducted during the screening procedure. Additional assessments included neuropsychological tests, neurobehavioral tests, and structural neuroimaging measurements; for a full overview of the study procedure, see the PPMI website (https://www.ppmi-info.org). The neurobehavioral assessments including the QUIP-S, GDS-15, and STAI-Y were conducted every six months during the first year and after that annually. Details on medication use at each of these visits were additionally documented.

\section{Statistical analysis}

We used Cox proportional hazards models to test the hypotheses that higher baseline depression and anxiety levels as well as their interaction with DA use are significant risk factors for the development of ICDs at follow-up visits. In addition, we set up an exploratory Cox proportional hazards model in which we included State and Trait STAI-Y scores separately to evaluate whether these carry different risks for ICD development. The fit of these two models was compared by means of the Akaike information criterion (AIC). In case of significant associations between any of the investigated factors and the dependent variables ICD risk, additional models were developed adjusting for the potential confounders Age, Gender, and UPDRS-motor score (as a proxy of PD symptom severity). We checked the proportional hazards assumption by assessing Schoenfeld's residuals, which showed that the assumption was met for all models. However, the linearity assumption was not consistently met, which was resolved by centering the continuous variables (i.e., GDS-15 and STAI-Y scores). Finally, we created reversed survival curves to illustrate the effect of baseline (total and Trait) STAI-Y scores on ICD development. All analyses were performed with $\mathrm{R}$ software version 4.0.5 using the "survival_3.2-10" and "AICcmodavg_2.31 " packages.

\section{RESULTS}

The final research sample consisted of 330 de novo PD patients (66.1\% male). Table 1 provides an overview of their demographic and clinical characteristics. Information about dopaminergic medication type usage was available for all participants: 106 patients $(32.1 \%)$ received DAs as their first DRT type, and 152 patients $(46.1 \%)$ received DAs at any point during the study period. Of the patients in our sample, $149(45.2 \%)$ developed ICDs across the course of the study, presenting (a combination of) the subtypes gambling $(n=14)$, hypersexuality $(n=41)$, buying $(n=30)$, eating $(n=49)$, and other compulsive behaviors (i.e., punding, hobbyism, or walkabout; $n=95$ ). The onset of these ICDs ranged from 3 until 96 months after the initial baseline visit, with an average of 34.54 months $(S D=24.74)$; the onset time distribution of our final sample is shown in Fig. 2.

\section{ICD risk factors}

Our Cox proportional hazards model revealed that higher STAI-Y scores were associated with an increased risk of developing an ICD at follow-up visits $(\mathrm{HR}=1.02,95 \% \mathrm{CI}=1.00-1.03), B=0.02$, Wald 
Table 1

Demographic and clinical characteristics at baseline for the patients included in our final sample $(N=330)$

\begin{tabular}{lcccc}
\hline Measure & $M$ & SD & Min & Max \\
\hline Age (years) & 61.48 & 9.51 & 33 & 84 \\
Education (years) & 15.58 & 2.94 & 5 & 26 \\
Disease duration (months) & 6.49 & 6.35 & 0 & 35 \\
UPDRS motor score & 21.18 & 9.11 & 4 & 51 \\
GDS-15 total score & 2.11 & 2.38 & 0 & 14 \\
STAI-Y total score & 63.53 & 18.10 & 40 & 137 \\
$\quad$ State subscore & 32.29 & 10.13 & 20 & 76 \\
$\quad$ Trait subscore & 31.24 & 9.24 & 20 & 63 \\
\hline
\end{tabular}

GDS-15, Geriatric Depression Scale; STAI-Y, State-Trait-Anxiety Inventory; UPDRS, Unified Parkinson's Disease Rating Scale.

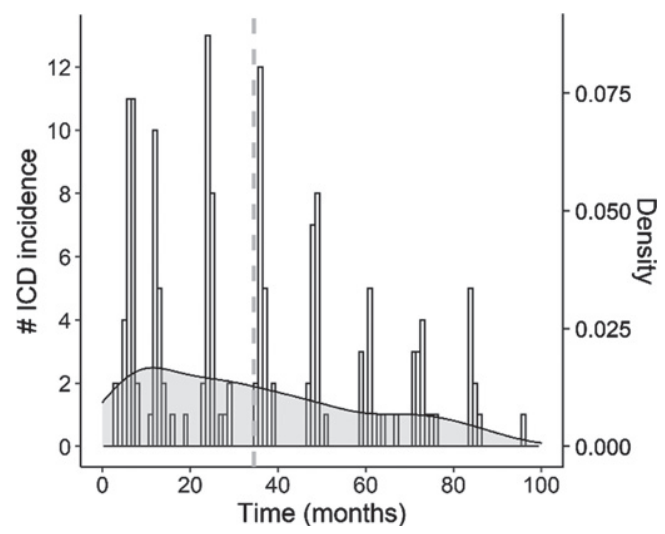

Fig. 2. Histogram with overlaid density plot showing the distribution of ICD onset times across patients in the study. The vertical dashed line indicates the average onset time $(M=34.54)$.

$\chi^{2}(1)=2.29, p=0.022$ (see Fig. 3). Results further showed that DAs were associated with a higher risk for ICDs $(\mathrm{HR}=1.46,95 \% \mathrm{CI}=1.02-2.10), B=0.38$, Wald $\chi^{2}(1)=2.04, p=0.042$. GDS-15 scores did not significantly predict ICD development $(B=-0.03$, Wald $\left.\chi^{2}(1)=-0.65, p=0.518\right)$, and neither did the interaction terms GDS-15 $\times$ DRT type and STAIY $\times$ DRT type $(p s>0.640)$.

An extended Cox model including the factors Age, Gender and UPDRS motor score showed that STAI-Y scores, but not DRT type ( $p=0.052)$, remained significantly associated with increased risk of developing an ICD $(\mathrm{HR}=1.02,95 \% \mathrm{CI}=1.00-1.03), B=0.02$, Wald $\chi^{2}(1)=2.44, p=0.014$. Results additionally showed that males carried an increased risk for developing an ICD compared to females ( $\mathrm{HR}=1.52$, $95 \% \mathrm{CI}=1.05-2.01), B=0.42$, Wald $\chi^{2}(1)=2.21$, $p=0.027$. None of the other factors were significant ( $p s>0.252$ ).

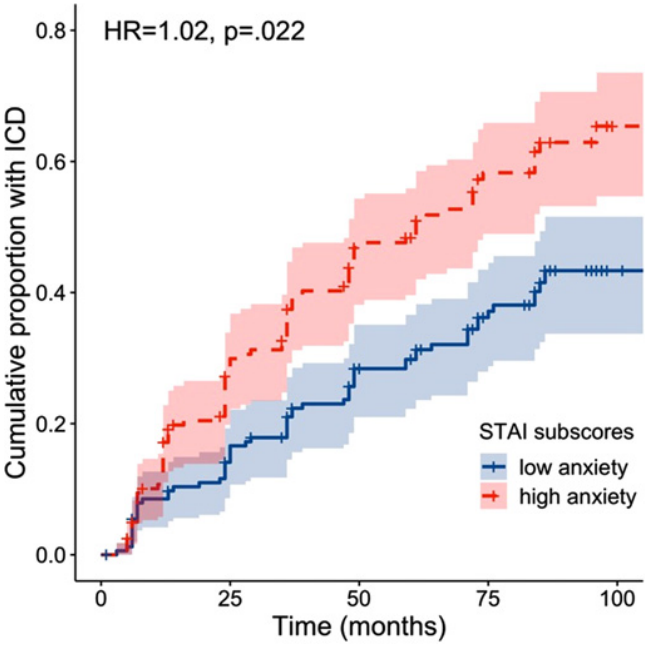

Fig. 3. Survival plot showing the cumulative proportion of patients with an ICD at each follow-up as a function of baseline anxiety. Note that baseline anxiety was dichotomized for visualization purposes into high and low anxiety by means of a median split on STAI-Y scores $($ median $=60.5$ ).

\section{Exploratory analysis of State-and Trait STAI-Y scores}

The exploratory Cox proportional hazards model revealed that higher Trait STAI-Y subscores were associated with an increased risk of developing an $\mathrm{ICD}(\mathrm{HR}=1.04,95 \% \mathrm{CI}=1.01-1.08), B=0.04$, Wald $\chi^{2}(1)=2.20, p=0.028$ (see Fig. 4). However, State STAI-Y subscores did not significantly predict ICD development, $B=0.00$, Wald $\chi^{2}(1)=-0.10$, $p=0.918$. Results for DRT type showed a similar pattern as in the model with total STAI-Y scores, yet this predictor was not significant, $B=0.36$, Wald $\chi^{2}(1)=1.91, p=0.056$. None of the other factors significantly predicted ICD development (all $p s>0.081$ ). Of note is that this model including the STAI-Y subscales did not show a better fit $(\mathrm{AICc}=1589.64)$ compared to the model with total STAI-Y scores $($ AICc $=1589.19)$. Finally, Trait STAI-Y subscores remained a significant predictor when adjusting for the factors Age, Gender and UPDRS motor score $(\mathrm{HR}=1.05,95 \% \mathrm{CI}=0.96-1.01$, $p=0.014)$.

\section{DISCUSSION}

This study examined the role of depression and anxiety, and the interaction of these factors with DRT use, for the development of ICDs in de novo PD patients. Almost half of the research sample 


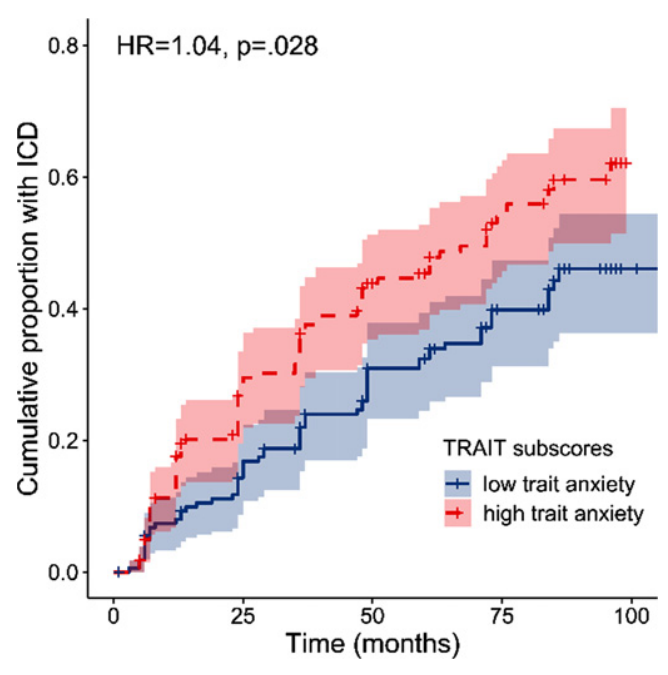

Fig. 4. Survival plot showing the cumulative proportion of patients with an ICD at each follow-up as a function of baseline trait anxiety. Note that baseline trait anxiety was dichotomized for visualization purposes into high and low trait anxiety by means of a median split on STAI-Y Trait scores (median $=29.5$ ).

developed an ICD over time, underlining the importance of identifying relevant risk factors in this patient population. We found that higher anxiety, and particularly trait anxiety, around the time of PD diagnosis is significantly associated with an increased risk for ICDs. Importantly, this effect was not confounded by age, gender, or PD symptom severity. In addition, we replicated the well-established finding that the use of DAs is associated with an increased risk for ICD development in people with PD. Finally, there was no significant effect for depression and no interaction between anxiety or depression with DRT type in the prediction of ICDs.

To our knowledge, this is the first study to show a temporal relationship between anxiety and ICD development in de novo PD patients. The finding that patients with higher anxiety levels around the time of PD diagnosis are more likely to develop ICDs during the course of the disease, indicates that anxiety precedes ICD development and should therefore be considered a relevant risk factor. Consequently, our study extends prior cross-sectional work which showed a general association between anxiety levels and ICDs in people with PD [21]. Around the time of PD diagnosis, patients might feel particularly anxious and experience excessive psychological distress, due to the uncertainty and drastic consequences that are associated with the disorder. Our results suggest that this high level of psychological distress might induce some kind of susceptibility for ICDs in these patients.
In healthy participants, increased psychological distress appears to be associated with worse self-control regarding behaviors such as eating or shopping [30]. These behaviors, expressed at a high rate and initially aimed at inducing a better feeling, might equally be present in de novo PD patients with high anxiety levels, potentially explaining the observed susceptibility for ICDs.

Furthermore, our results revealed that in particular higher trait anxiety, but not state anxiety, around the time of PD diagnosis was a significant risk factor for ICD development. This differential effect between the two facets of anxiety is in line with observations from a cross-sectional study that also found a significant association of higher trait but not state scores with ICDs in people with PD [31]. Considering the relatively permanent and general nature of trait anxiety, it appears reasonable that it might also be of greater relevance for the development of ICDs, as compared to the temporary state of feeling anxious. Although our model with the two separate subscales was not significantly better in predicting ICD presence, as compared to the model including total STAI-Y scores, it might be warranted to differentiate between state and trait anxiety measures when further investigating its predictive value for ICDs.

In contrast to anxiety, baseline depression levels were not found to be a significant risk factor for ICD development in the current study. This finding is inconsistent with a recent study using PPMI data which showed that a diagnosis of depression shortly after PD diagnosis is associated with an increased ICD risk later in the disease [18]. A possible explanation for this inconsistency might be related to the definition of depression in both studies. More specifically, the researchers [18] classified patients as depressed or not, with the definition of depression being either a GDS-15 score above the cut-off of 7 or an existing diagnosis of as depressed and already received antidepressants. In contrast, the current study considered depressive symptoms as a continuous variable based on GDS-15 scores and therefore did not separately consider treated depressed patients. Although this does not fully explain the conflicting results, it underlines the importance of taking also treated depression into account when investigating risk factors for ICDs in PD. Another potential explanation for the finding that baseline depression is not significantly associated with ICD development, is that depression might represent a greater risk factor at a more advanced PD stage as compared to the time around the initial diagnosis. Baseline 
depression scores were relatively low (GDS-15 scores: $M=2.11 \pm 2.38$ ) in this study compared to depression rates which are usually reported in people with PD (e.g., GDS-15 scores: $M=4.20 \pm 4.00$ in Weintraub et al. (2007) [32]). Interestingly, a recent PPMI study showed an increase in depressive symptoms in the first five years after the initial PD diagnosis [33]. Future research should therefore focus on the predictive value of depression for ICD development around a more advanced disease stage. Lastly, it must also be considered that depression may occur in response to ICDs in some patients. This not only seems reasonable given the negative consequences that are commonly associated with ICDs, but it would also be in line with multiple studies reporting higher depression rates in patients with ICDs as compared to those without $[15,19]$.

Given that depression and anxiety share certain symptoms and frequently co-occur in PD [34], one may argue that these overlapping aspects (rather than non-overlapping aspects) could drive the present results. However, the validated instruments used to assess anxiety and depression in the PPMI study and therefore the present work have specifically been designed to reduce the overlap and obtain a pure measure of anxiety and depression as much as possible. Furthermore, we found that only STAI-Y scores but not GDS-15 scores significantly predicted future ICD development. It therefore seems unlikely that the shared aspects of anxiety and depression would underlie the present findings.

The current study found no significant interactions between the investigated psychological factors and DRT in the prediction of ICDs. This is not in line with the model described by Deleaney et al. (2012) [23], which assumes that DA treatment multiplies the susceptibility that arises from psychological factors for the occurrence of ICDs in people with PD. Our results, however, suggest that patients with increased anxiety levels around the time of PD diagnosis do not face an increased ICD risk when treated with DAs, as compared to patients with similar anxiety levels who do not receive DA treatment. In line with previous findings [18], we also found no interaction between DRT and depression in the prediction of ICDs. Taken together, our current and previous findings suggest that the multiplier model should be refined as DAs may interact with some, but not all psychosocial factors.

With regard to the clinical significance of the present results, it should be noted that the hazard ratio for DA use (1.46) was larger in comparison to that for anxiety (1.02). While our findings suggest that anxiety symptoms at the time of PD diagnosis could be useful in the prediction of future ICD development, they more so emphasize the importance of DA use in this context [cf. 5-8]. Consequently, in clinical practice the type of dopaminergic medication should continuously be considered when evaluating the risk for ICDs in people with PD from the moment they first start DRT. Notwithstanding this relatively strong predictive role of DA, our present findings suggest that in particular for de novo patients who have not started any medication yet, it could be informative for clinicians to assess anxiety symptoms to gauge potential ICD risk.

A notable strength of this study is the prospective longitudinal research design, which allowed us to investigate the temporal relationship of the two psychological factors, anxiety and depression, with ICD development. Another strength is the time-dependent nature of the DRT variable. Given that medication regimes are frequently adjusted over the course of the disease depending on their effectiveness and/or negative side effects, it is important to consider these changes when investigating its role in ICD development. Moreover, the statistical approach that controlled for confounding factors such as age, gender, and motor symptom severity, further strengthens the findings from this study by demonstrating stability of the established associations. A limitation of this study is that impulsivity and neuropsychiatric symptoms were assessed by means of self-report measures. Although the QUIP-S, GDS-15 and STAI$\mathrm{Y}$ have been recommended for the use in people with PD [25-29], future studies should consider also including an objective clinical assessment to validate diagnoses of these symptoms. Another limitation of this study might be the lack of differentiation regarding DRT types beyond DA versus other types. The latter category comprised several different DRT types including levodopa, MAO-B inhibitors, and amantadine. Moreover, also DAs can be further differentiated into subtypes, which may be differently associated with ICD risk. For instance, a prior study [35] showed that ICD prevalence was higher in PD patients receiving non-ergolinic oral DAs, such as pramipexole and ropinirole, as compared to rotigotine, a transdermal non-ergolinic DA [35]. Future research should further investigate these differential effects of DAs and other DRT subtypes in interaction with psychological predictors of ICDs, such as anxiety, in order to extend the current findings. Furthermore, future research may also evaluate whether baseline (trait) anxiety is 
a stable predictor across patients with different PD motor subtypes. Specifically, the comparison of the 'tremor-dominant' and 'postural instability and gait dysfunction' subtypes appear relevant in this context, since primarily the latter has been associated with increased anxiety prevalence [36].

In summary, our study revealed that de novo PD patients with higher anxiety levels, but not higher depression levels, are at increased risk of developing ICDs during the course of the disease. We found that in particular higher trait anxiety is associated with an increased risk for ICD development. We also replicated prior findings that DA use is a risk factor for development of ICDs in people with PD. As we did not observe significant interactions between psychological symptoms and DA use in the prediction of ICDs, it seems that these may be independent risk factors. In addition to confirming the importance of managing dopaminergic medication in the context of ICDs throughout the disease course, our findings highlight the need for screening (trait) anxiety symptoms at the time of PD diagnosis. This may allow for the early identification and targeted treatment of anxiety symptoms relatively early on, which could consequently reduce or delay ICD development. Ultimately, early screening could benefit informed treatment decisions, reduce secondary consequences of ICDs, and increase quality of life.

\section{ACKNOWLEDGMENTS}

PPMI, a public-private partnership, is funded by the Michael J. Fox Foundation for Parkinson's Research funding partners 4D Pharma, Abbvie, Acurex Therapeutics, Allergan, Amathus Therapeutics, ASAP, Avid Radiopharmaceuticals, Bial Biotech, Biogen, BioLegend, Bristol-Myers Squibb, Calico, Celgene, Dacapo Brain Science, Denali, The Edmond J. Safra Foundaiton, GE Healthcare, Genentech, GlaxoSmithKline, Golub Capital, Handl Therapeutics, Insitro, Janssen Neuroscience, Lilly, Lundbeck, Merck, Meso Scale Discovery, Neurocrine Biosciences, Pfizer, Piramal, Prevail, Roche, Sanofi Genzyme, Servier, Takeda, Teva, UCB, Verily, and Voyager Therapeutics.

\section{CONFLICT OF INTEREST}

The authors have no conflict of interest to report.

\section{REFERENCES}

[1] Averbeck BB, O'Sullivan SS, Djamshidian A (2014) Impulsive and compulsive behaviors in Parkinson's disease. Annu Rev Clin Psychol 10, 553-580.

[2] Schapira AH, Chaudhuri KR, Jenner P (2017) Non-motor features of Parkinson disease. Nat Rev Neurosci 18, 435450.

[3] Weintraub D, Claassen DO (2017) Impulse control and related disorders in Parkinson's disease. Int Rev Neurobiol 133, 679-717.

[4] Gatto EM, Aldinio V (2019) Impulse control disorders in Parkinson's disease. A brief and comprehensive review. Front Neurol 10, 351.

[5] Ambermoon P, Carter A, Hall WD, Dissanayaka NN, O'Sullivan JD (2011) Impulse control disorders in patients with Parkinson's disease receiving dopamine replacement therapy: evidence and implications for the addictions field. Addiction 106, 283-293.

[6] Corvol JC, Artaud F, Cormier-Dequaire F, Rascol O, Durif F, Derkinderen P, Marques AR, Bourdain F, Brandel JP, Pico F, Lacomblez L, Bonnet L, Brefel-Courbon C, Ory-Magne F, Grabli F, Klebe F, Mangone G, You H, Mesnage V, Lee PC, Brice A, Vidailhet M, Elbaz A (2018) Longitudinal analysis of impulse control disorders in Parkinson disease. Neurology 91, e189-e201.

[7] Erga AH, Alves G, Larsen JP, Tysnes OB, Pedersen KF (2017) Impulsive and compulsive behaviors in Parkinson's disease: the Norwegian ParkWest study. J Parkinsons Dis 7, 183-191.

[8] Weintraub D, Koester J, Potenza MN, Siderowf AD, Stacy M, Voon V, Whetteckey J, Wunderlich GR, Lang AE (2010) Impulse control disorders in Parkinson disease: a crosssectional study of 3090 patients. Arch Neurol 67, 589-595.

[9] Jakuszkowiak-Wojten K, Landowski J, Wiglusz MS, Cubała WJ (2015) Impulsivity in anxiety disorders: a critical review. Psychiatr Danub 27, 452-455.

[10] Moustafa AA, Tindle R, Frydecka D, Misiak B (2017) Impulsivity and its relationship with anxiety, depression and stress. Compr Psychiatry 74, 173-179.

[11] Faivre F, Joshi A, Bezard E, Barrot M (2019) The hidden side of Parkinson's disease: studying pain, anxiety and depression in animal models. Neurosci Biobehav Rev 96, 335-352.

[12] Aarsland D, Marsh L, Schrag A (2009) Neuropsychiatric symptoms in Parkinson's disease. Mov Disord 24, 2175 2186.

[13] Martinez-Martin P, Rodriguez-Blazquez C, Forjaz MJ, Frades-Payo B, Agüera-Ortiz L, Weintraub D, Riesco A, Kurtis MM, Chaudhuri KR (2015) Neuropsychiatric symptoms and caregiver's burden in Parkinson's disease. Parkinsonism Relat Disord 21, 629-634.

[14] Yamanishi T, Tachibana H, Oguru M, Matsui K, Toda K, Okuda B, Oka N (2013) Anxiety and depression in patients with Parkinson's disease. Intern Med 52, 539-545.

[15] Callesen MB, Weintraub D, Damholdt MF, Møller A (2014) Impulsive and compulsive behaviors among Danish patients with Parkinson's disease: prevalence, depression, and personality. Parkinsonism Relat Disord 20, 22-26.

[16] Joutsa J, Martikainen K, Vahlberg T, Voon V, Kaasinen V (2012) Impulse control disorders and depression in Finnish patients with Parkinson's disease. Parkinsonism Relat Disord 18, 155-160.

[17] Eisinger RS, Ramirez-Zamora A, Carbunaru S, Ptak B, Peng-Chen Z, Okun MS, Gunduz A (2019) Medications, 
deep brain stimulation, and other factors influencing impulse control disorders in Parkinson's disease. Front Neurol 10, 86.

[18] Marín-Lahoz J, Sampedro F, Martinez-Horta S, Pagonabarraga J, Kulisevsky J (2019) Depression as a risk factor for impulse control disorders in Parkinson disease. Ann Neurol 86, 762-769.

[19] Martini A, Dal Lago D, Edelstyn NM, Grange JA, Tamburin S (2018) Impulse control disorder in Parkinson's disease: a meta-analysis of cognitive, affective, and motivational correlates. Front Neurol 9, 654.

[20] Pontieri FE, Assogna F, Pellicano C, Cacciari C, Pannunzi S, Morrone A, Danese E, Caltagirone C, Spalletta G (2015) Sociodemographic, neuropsychiatric and cognitive characteristics of pathological gambling and impulse control disorders NOS in Parkinson's disease. Eur Neuropsychopharmacol 25, 69-76.

[21] Voon V, Sohr M, Lang AE, Potenza MN, Siderowf AD, Whetteckey J, Weintraub D, Wunderlich R, Stacy M (2011) Impulse control disorders in Parkinson disease: a multicenter case-control study. Ann Neurol 69, 986-996.

[22] Leroi I, Ahearn DJ, Andrews M, McDonald KR, Byrne EJ, Burns A (2011) Behavioural disorders, disability and quality of life in Parkinson's disease. Age Ageing 40, 614-621.

[23] Delaney M, Leroi I, Simpson J, Overton PG (2012) Impulse control disorders in Parkinson's disease: a psychosocial perspective. J Clin Psychol Med Settings 19, 338-346.

[24] Parkinson Progression Marker Initiative (2011) The Parkinson Progression Marker Initiative (PPMI). Prog Neurobiol 95, 629-635.

[25] Weintraub D, Hoops S, Shea JA, Lyons KE, Pahwa R, Driver-Dunckley ED, Adler CH, Potenza MN, Miyasaki J, Siderowf AD, Duda JE, Hurtig HI, Colcher A, Horn SS, Stern MB, Voon V (2009) Validation of the questionnaire for impulsive-compulsive disorders in Parkinson's disease. Mov Disord 24, 1461-1467.

[26] Probst CC, Winter LM, Möller B, Weber H, Weintraub D, Witt K, Deuschl G, Katzenschlager R, van Eimeren T (2014) Validation of the questionnaire for impulsive-compulsive disorders in Parkinson's disease (QUIP) and the QUIPrating scale in a German speaking sample. J Neurol 261, 936-942.

[27] Weintraub D, Oehlberg KA, Katz IR, Stern MB (2006) Test characteristics of the 15-item geriatric depression scale and Hamilton depression rating scale in Parkinson disease. Am J Geriatr Psychiatry 14, 169-175.
[28] Mondolo F, Jahanshahi M, Grana A, Biasutti E, Cacciatori E, Di Benedetto P (2007) Evaluation of anxiety in Parkinson's disease with some commonly used rating scales. Neurol Sci 28, 270-275.

[29] Yang HJ, Ahn JH, Lee J, Lee WK, Lee JH, Kim Y (2019) Measuring anxiety in patients with early-stage Parkinson's disease: Rasch analysis of the State-Trait Anxiety Inventory. Front Neurol 10, 49.

[30] Tice DM, Bratslavsky E, Baumeister RF (2001) Emotional distress regulation takes precedence over impulse control: If you feel bad, do it! J Pers Soc Psychol 80, 53.

[31] Sáez-Francàs N, Andrés GM, Ramírez N, de Fabregues O, Álvarez-Sabín J, Casas M, Hernández-Vara J (2016) Clinical and psychopathological factors associated with impulse control disorders in Parkinson's disease. $\mathrm{Neu}$ rología (English Edition) 31, 231-238.

[32] Weintraub D, Xie S, Karlawish J, Siderowf A (2007) Differences in depression symptoms in patients with Alzheimer's and Parkinson's diseases: evidence from the 15-item Geriatric Depression Scale (GDS-15). Int J Geriatr Psychiatry 22, 1025-1030

[33] Weintraub D, Caspell-Garcia C, Simuni T, Cho HR, Coffey CS, Aarsland D, Alcalay RN, Barrett MJ, Chahine LM, Eberling J, Espay AJ, Hamilton J, Hawkins KA, Leverenz J, Litvan I, Richard I, Rosenthal LS, Siderowf A, York M, Parkinson's Progression Markers Initiative (2020) Neuropsychiatric symptoms and cognitive abilities over the initial quinquennium of Parkinson disease. Ann Clin Transl Neurol 7, 449-461.

[34] Rutten S, Ghielen I, Vriend C, Hoogendoorn AW, Berendse HW, Leentjens AF, van der Werf YD, Smit JH, van den Heuvel OA (2015) Anxiety in Parkinson's disease: Symptom dimensions and overlap with depression and autonomic failure. Parkinsonism Relat Disord 21, 189-193.

[35] Garcia-Ruiz PJ, Castrillo JCM, Alonso-Canovas A, Barcenas AH, Vela L, Alonso PS, Mata M, Gonzales NO, Fernandez IM (2014) Impulse control disorder in patients with Parkinson's disease under dopamine agonist therapy: a multicentre study. J Neurol Neurosurg Psychiatry 85, 840844.

[36] Dissanayaka NN, Sellbach A, Matheson S, O'Sullivan JD, Silburn PA, Byrne GJ, Marsh R, Mellick GD (2010) Anxiety disorders in Parkinson's disease: prevalence and risk factors. Mov Disord 25, 838-845. 